\title{
Mifepristone in Fibroids: An Alternative!
}

\author{
${ }^{1}$ Sunita Lamba, ${ }^{2}$ Aabha Singh, ${ }^{3}$ Priyankur Roy, ${ }^{4}$ Ruchika Garg
}

\section{ABSTRACT}

Introduction: Medical management is a keystone in managing symptomatic fibroids. Availability of an effective, safe, and costeffective medical treatment should always be considered as an alternative to reduce the cost, morbidity, and impact of avoidable major surgeries.

Objective: To study the effect of $10 \mathrm{mg}$ Mifepristone on symptomatic fibroids.

Materials and methods: This prospective study was conducted at a tertiary care center in Raipur. Fifty women of the reproductive age group with symptomatic uterine fibroids with normal liver and kidney function tests were recruited. A total of $10 \mathrm{mg}$ of oral Mifepristone was administered daily for 3 months. Monthly symptomatic assessment, ultrasonography, and biochemical parameters were done to assess changes.

Results: There was significant reduction in menstrual blood loss (MBL) and associated symptoms. Amenorrhea occurred in 100\% of women after 3 months of treatment. The fibroid volume and mean uterine size reduced by 26.2 and $26.99 \%$ respectively. Mean hemoglobin levels increased by $1.93 \mathrm{gm} / \mathrm{dL}$. Observed side effects were few and tolerable.

Conclusion: Mifepristone is an effective and safe alternative for the management of leiomyoma.

Keywords: Fibroid volume, Leiomyoma, Mean uterine size, Medical management, Mifepristone, Symptomatic fibroids.

How to cite this article: Lamba S, Singh A, Roy P, Garg R. Mifepristone in Fibroids: An Alternative!. J South Asian Feder Obst Gynae 2018;10(1):29-32.

Date of received: 15 January 2018

Date of acceptance: 25 February 2018

Date of publication: May 2018

Source of support: Nil

Conflict of interest: None

\footnotetext{
${ }^{1}$ Clinical Fellow, ${ }^{2}$ Professor, ${ }^{3}$ Tutor, ${ }^{4}$ Associate Professor

${ }^{1}$ Department of Obstetrics and Gynecology, Institute of Kidney Diseases and Research Centre, Ahmedabad, Gujarat, India

2Department of Obstetrics \& Gynecology, Pt. Jawahar Lal Nehru Memorial Medical College, Raipur, Chhattisgarh, India

${ }^{3}$ Department of Obstetrics and Gynecology, North Bengal Medical College \& Hospital, Siliguri, West Bengal, India

${ }^{4}$ Department of Obstetrics \& Gynecology, Sarojini Naidu Medical College, Agra, Uttar Pradesh, India

Corresponding Author: Sunita Lamba, Clinical Fellow Department of Obstetrics and Gynecology, Institute of Kidney Diseases and Research Centre, Ahmedabad, Gujarat, India Phone: +918740834555, e-mail: sunita.lamba011@gmail.com
}

\section{INTRODUCTION}

Uterine fibroids are the most common benign tumors of women. They commonly present with menstrual disturbances, pain, pressure symptoms, and fertility problems. The disabling symptoms and absence of effective medical therapy attribute to high rates of surgical management of fibroids. It is a common indication for hysterectomy leading to financial burden. Though minimal invasive methods like uterine artery embolization, high intensity focused ultrasound, and magnetic resonance-guided focused ultrasound are available, still they are beyond the reach of most women due to high expertise required and cost. Medical management like gonadotropin-releasing hormone (GnRH) analog and Danazol are expensive and have a number of side effects. Thus, the search for an acceptable, safe, and effective nonsurgical treatment option for symptomatic leiomyoma is of considerable clinical and public health importance.

Mifepristone is a synthetic derivative of norethindrone with antiprogesterone activity. ${ }^{1}$ Various studies have suggested its use in fibroids. ${ }^{2}$ It has been found to improve the quality-of-life in symptomatic women with fibroids. ${ }^{3}$ Our study was conducted to assess the effects of Mifepristone on symptomatic fibroids and its side effects.

\section{MATERIALS AND METHODS}

Fifty women with symptomatic fibroids who presented to the gynecology outpatient department in a tertiary care center in Raipur were included in the study. It was a prospective interventional study. The study was taken up after obtaining Institutional Ethical Committee clearance.

Study Outcome: The present study was designed to evaluate the effectiveness of $10 \mathrm{mg}$ Mifepristone on symptomatic fibroids.

Study Design: Prospective interventional study.

Study Population: All women with symptomatic fibroids, fulfilling the inclusion criteria were enrolled into the study.

Study Period: Two years.

Informed Consent: All the women and the attenders gave written informed consent before administration of Mifepristone. 


\section{Inclusion Criteria}

- Women with symptomatic fibroids and willing for regular follow-up

- Women in the reproductive age group.

\section{Exclusion Criteria}

- Pregnant women

- Women desirous of pregnancy

- Breastfeeding women

- History of intake of hormonal therapy in the previous 3 months

- Diagnosed or suspected ovarian, cervical, or uterine malignancy

- Presence of medical ailments like liver disorders, renal disorders, heart disease, or adrenal disorders

- Pelvic inflammatory disease or other adnexal pathologies

- Psychiatric disorders

- Sickle cell anemia

- Bleeding disorders

- Woman necessitating early surgical intervention were also excluded.

Informed consent was obtained from all recruited women and their attenders. Women were assessed for fibroid-related symptoms on a visual analog score. Baseline pelvic ultrasound and blood parameters, such as hemoglobin and liver and kidney function tests were done. Women were followed monthly during the treatment period for 3 months. Monthly symptomatic score was done on the visual analog score. Pelvic ultrasound and blood parameters were again repeated after 3 months of initiation of treatment. Side effects were noted. Women were followed up until 6 months for noting any recurrence of symptoms.

\section{Visual Analog Scale used for Symptomatology}

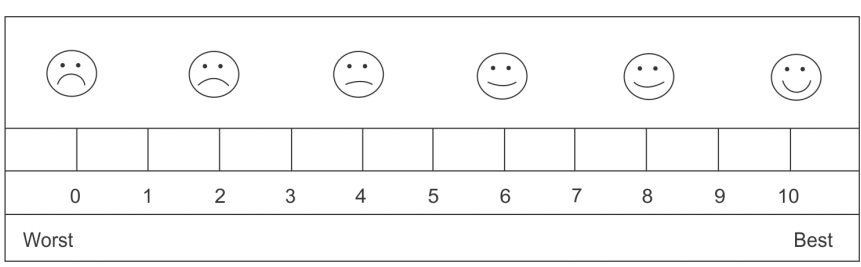

Table 1: Profile of women in the study group

\begin{tabular}{llll}
\hline Characteristics & Mean & SD & Range \\
\hline Age (years) & 38.92 & 5.75 & $24-48$ \\
Parity & 2.26 & 3.34 & $1-5$ \\
Body mass index $\left(\mathrm{kg} / \mathrm{m}^{2}\right)$ & 25.28 & 3.24 & $17-32$ \\
Uterine volume (cc) & 204.03 & 113.12 & $85-600$ \\
Fibroid volume (cc) & 36.56 & 41.39 & $4-128$ \\
\hline
\end{tabular}

SD: Standard deviation

\section{Statistical Methods}

Statistical analysis was carried out using commercial software Statistical Package for the Social Sciences version 20. The descriptive measures like mean, median, and standard deviation for continuous variables were obtained. Frequencies and percentages were calculated for all categorical variables.

\section{RESULTS}

All 50 women came for regular follow-up for the entire period of 6 months from the initiation of treatment. The baseline characteristics of women enrolled in the study are shown in Table 1.

The various symptoms of women and their improvement after Mifepristone therapy are shown in Table 2. Severity of the symptoms on visual analog score decreased satisfactorily in the majority of women. Symptomatic relief was significant at end of 3 months. Cessation of the menstruation was the most prominent finding in Mifepristone-treated women. Amenorrhea occurred in $90 \%$ of women by the end of first month and $100 \%$ of the women at the end of third month of treatment. Due to amenorrhea, $100 \%$ of women were relieved of dysmenorrhea completely. Menstrual blood loss decreased significantly ( $p$-value 0.0001 ) even after 1 month of therapy. About $96 \%$ women regained their menses after cessation of drug within 6 weeks. Menstrual blood loss remained significantly low in $87 \%$ of women in follow-up until 6 months of initiation of therapy.

In the present study, the mean uterine volume was reduced significantly ( $p$-value 0.006 ) after 3 months of treatment. Reduction in volume was also achieved in first month $(8.93 \%$ decrease) and second month $(17.41 \%$ decrease) of therapy, but it was only significant after 3 months of treatment (Table 3).

Reduction in leiomyoma volume by $26.2 \%$ was observed at the end of therapy. However, reduction in fibroid volume was not significant statistically. There was no significant change in endometrial thickness (Table 3). In six women, fibroid volume did not change, but symptomatic relief was present.

Table 2: Distribution of women according to complete resolution of symptoms

\begin{tabular}{llll}
\hline Symptoms & Baseline & 3 months & 6 months \\
\hline $\begin{array}{l}\text { Menstrual } \\
\text { disturbances }\end{array}$ & 45 & 0 & 6 \\
Dysmenorrhea & 38 & 0 & 1 \\
Pelvic pain & 30 & 19 & 14 \\
Backache & 18 & 12 & 10 \\
Urinary complaints & 15 & 9 & 5 \\
\hline
\end{tabular}


Table 3: Effect on ultrasound parameters

\begin{tabular}{lllll}
\hline & \multicolumn{3}{c}{ At 3} \\
Parameters & Baseline & months & \% change & p-value \\
\hline Uterine volume & $204.03 \pm$ & $148.96 \pm$ & 26.99 & 0.006 \\
(cc) & 113.12 & 86.88 & & \\
Fibroid volume & $36.56 \pm$ & $26.99 \pm$ & 26.2 & 0.200 \\
(cc) & 41.39 & 32.91 & & \\
$\begin{array}{l}\text { Endometrial } \\
\text { thickness }(\mathrm{mm})\end{array}$ & $7.00 \pm$ & $7.19 \pm$ & 2.5 & 0.722 \\
\hline
\end{tabular}

Table 4: Effect on hematological parameters

\begin{tabular}{|c|c|c|c|}
\hline Parameters & Baseline & After therapy & $p$-value \\
\hline Hemoglobin (gm/dL) & $9.51 \pm 1.31$ & $11.44 \pm 0.78$ & 0.0001 \\
\hline Serum bilirubin (mg/dL) & $0.38 \pm 0.16$ & $0.42 \pm 0.16$ & 0.30 \\
\hline $\begin{array}{l}\text { Alanine aminotransferase } \\
(\mathrm{U} / \mathrm{L})\end{array}$ & $19.4 \pm 6.55$ & $19.62 \pm 5.64$ & 0.85 \\
\hline $\begin{array}{l}\text { Aspartate } \\
\text { aminotransferase }(\mathrm{U} / \mathrm{L})\end{array}$ & $18.86 \pm 6.66$ & $19.9 \pm 6.39$ & 0.42 \\
\hline Blood urea (mg) & $17.50 \pm 7.87$ & $16.76 \pm 4.18$ & 0.55 \\
\hline Serum creatinine $(\mathrm{mg})$ & $0.85 \pm 0.22$ & $0.82 \pm 0.13$ & 0.406 \\
\hline
\end{tabular}

Mean hemoglobin level increased by $1.93 \mathrm{gm} / \mathrm{dL}$ (p-value 0.001) after therapy. Significant increase was reported even after the second month of therapy. There was no negative effect on renal function tests (Table 4). In three women, mild elevation of liver transaminase was observed at end of 3 months, but was of no clinical significance. Observed values of liver transaminase were 50 to $55 \mathrm{IU}$ in these women (normal value: 46 and 49 IU for the aspartate aminotransferase and alanine transferase respectively).

Other observed side effects included hot flushes, which were noted in $3(6 \%)$ women, and $2(4 \%)$ women complained of mild joint pains. Potential side effects like nausea, vomiting, diarrhea, headache, weight gain, and loss of libido were not seen in the present study.

After completion of treatment, 42 (84\%) women remained relatively symptomless until the next 3 months

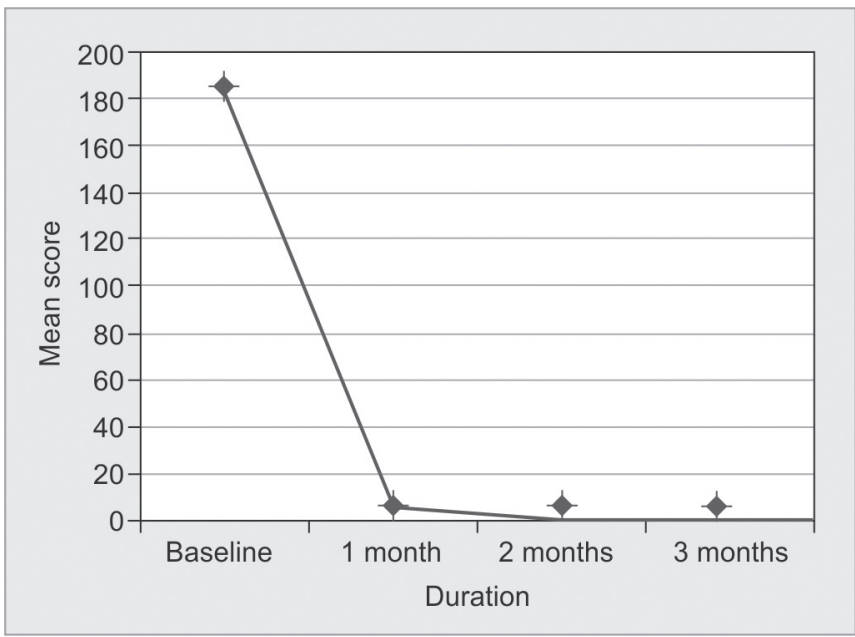

Graph 1: Effect of therapy on MBL on PBAC of follow-up. The effect of therapy on MBL on pictorial blood loss assessment chart (PBAC) is shown in Graph 1. Other forms of medical management like GnRH analogs were required in three women. Surgical intervention was required in five women. Hysterectomy was performed in three women for recurrence of menorrhagia and in another one woman for persistence of severe dysmenorrhea and pelvic pain. Myomectomy was required in one woman for no significant reduction in the size of abdominal lump.

\section{DISCUSSION}

There are various modalities for the management of fibroid uterus like expectant management, medical therapy, conventional surgical options, and newer less invasive approaches. The choice of the procedure depends on many factors including age, parity, fertility, severity of symptoms, size, number and location of fibroids, and desire for uterine preservation.

The present study has shown that 3 months of treatment with $10 \mathrm{mg}$ Mifepristone effectively improved fibroid-associated symptoms, such as bleeding, dysmenorrhea, pain, pressure symptoms, improvement in hemoglobin levels, and reduction in uterine and fibroid size. A $10 \mathrm{mg}$ dose is as effective as $50 \mathrm{mg}$ with lesser side effects. ${ }^{4}$

Mifepristone-induced reduction of menstrual bleeding and increase in hemoglobin level are the most important and useful observations of our study. Amenorrhea, in our study, was observed in 100\% women. It was in higher percentage as compared with other studies using similar dose and duration. ${ }^{1}$ These results are comparable with higher dose $(50 \mathrm{mg})$ administered for 3 months. ${ }^{4}$ Occurrence of amenorrhea was responsible for improvement of hemoglobin levels. Dysmenorrhea was also relieved completely in all women as a consequence of amenorrhea. In another study, Bagaria et $\mathrm{al}^{1}$ reported complete relief of dysmenorrhea in $80 \%$ of women. Pelvic pain was relieved in $36.6 \%$ women in our study. Eisinger et al ${ }^{5}$ reported pain relief in $41.6 \%$ women and they used ultralow dose of Mifepristone for 6 months. Prevalence and severity of backache and urinary complaints also decreased with Mifepristone. Similarly, symptomatic relief was reported by other studies. ${ }^{4-6}$

In our study, there was remarkable reduction in the uterine size and fibroid volume along with symptomatic response. Shikha et $\mathrm{al}^{7}$ showed 3 months treatment of $25 \mathrm{mg}$ Mifepristone effectively controls bleeding, reduces the uterine and myoma volume, and thus, can help avoid blood transfusion and hysterectomy in a number of symptomatic myoma cases. Symptomatic 
relief and reduction in size were less when dose of drug was reduced below $10 \mathrm{mg} .{ }^{6}$ Kulshrestha et $\mathrm{al}^{8}$ proved that Mifepristone (10 and $25 \mathrm{mg}$ ) caused symptomatic relief with more than $90 \%$ reduction in MBL. Greater myoma size reduction occurred with $25 \mathrm{mg}$ dose. Amenorrhea was developed in 90 to $95 \%$ patients, which was reversible.

After treatment, $1.93 \mathrm{gm} / \mathrm{dL}$ rise in hemoglobin level was noted. Improvement in hemoglobin was also reported in other studies. ${ }^{1,4}$ Elevation of liver transaminase was observed in three women $(6 \%)$ and was comparable with the results of other studies. ${ }^{2}$ This minimal elevation had no clinical significance. Bagaria et $\mathrm{al}^{1}$ did not report any alteration in liver enzymes. Kidney function tests remained unaltered.

Hot flushes were observed in three women and joint pain in two women. These side effects were relieved after stoppage of the drug. Carbonell et $\mathrm{al}^{2}$ also reported side effects in their study. In two Indian studies, no general side effects were reported., ${ }^{1,6}$ This short-term treatment with Mifepristone in our study was well tolerated and no serious side effects were noted.

\section{CONCLUSION}

Mifepristone can be considered as an effective alternative for management of fibroids. Lack of general and biochemical side effects together with excellent symptomatic relief confirm its suitability for management of fibroids and reducing major surgical procedures. However, long-term, large multicentric randomized controlled trials are required to decide dose, duration, long-term safety of drug and its effect on fertility.

\section{REFERENCES}

1. Bagaria M, Suneja A, Vaid NB, Guleria K, Mishra K. Low dose Mifepristone in treatment of uterine leiomyomas: a randomized double blind placebo controlled clinical trial. Aust NZ J Obstet Gynecol 2009 Feb;49(1):77-83.

2. Cabonell Esteve JL, Ascota R, Heredia B, Perez Y, Castaneda MC, Hernendez AV. Mifepristone for the treatment of uterine leiomyomas: a randomized controlled trial. Obstet Gynecol 2008 Nov;112(5):1029-1036.

3. Feng C, Meldrum S, Fiscella K. Improved quality of life is partly explained by fewer symptoms after treatment of fibroids with Mifepristone. Int J Gynaecol Obstet 2010 May;109(2):121-124.

4. Engman M, Granberg S, Williams AR, Meng CX, Lalitkumar PG, Gemzell-Danielsson K. Mifepristore for treatment of uterine leiomyoma. A prospective randomized placebo controlled trial. Hum Reprod 2009 Aug;24(8):1870-1879.

5. Eisinger SH, Fiscella J, Bonfiglio T, Meldrum S, Fiscella K. Open-label study of ultra low-dose Mifepristone for the treatment of uterine leiomyomata. Eur J Obstet Gynecol 2009 Oct;146(2):215-218.

6. Mukherjee S, Chakraborty SA. Study evaluating the effect of mifepristone (RU-486) for the treatment of leiomyomata uteri. Niger Med J 2011 Jul;52(3):150-152.

7. Shikha S, Neeru G, Ekta S, Mathur AS, Garima G. Effect of mifepristone $(25 \mathrm{mg})$ in treatment of uterine myoma in perimenopausal woman. J Midlife Health 2013 Jan-Mar;4(1):22-26.

8. Kulshrestha V, Kriplani A, Agarwal N, Sareen N, Garg P, Hari S, Thulkar J. Low dose mifepristone in medical management of uterine leiomyoma-an experience from a tertiary care hospital from north India. Indian J Med Res 2013 Jun; 137(6):1154-1162. 